\title{
Comparison between Golden Ball Meta-heuristic, Evolutionary Simulated Annealing and Tabu Search for the Traveling Salesman Problem
}

\author{
Eneko Osaba \\ University of Deusto \\ Av. Universidades, 24 \\ Bilbao, Spain \\ e.osaba@deusto.es \\ Pedro López-García \\ University of Deusto \\ Av. Universidades, 24 \\ Bilbao, Spain \\ p.lopez@deusto.es
}

\author{
Roberto Carballedo \\ University of Deusto \\ Av. Universidades, 24 \\ Bilbao, Spain \\ roberto.carballedo@deusto.es \\ Fernando Diaz \\ University of Deusto \\ Av. Universidades, 24 \\ Bilbao, Spain \\ fernando.diaz@deusto.es
}

\begin{abstract}
The Golden Ball is a multi-population meta-heuristic based on soccer concepts. It was first designed to solve combinatorial optimization problems. Until now, it has been tested with different kind of problems, but its efficiency has only been compared with some classical algorithms, such as different kind of Genetic Algorithms and Distributed Genetic Algorithms. In this work, the performance of the Golden Ball is compared with the ones obtained by two famous and widely used techniques: an Evolutionary Simulated Annealing and a Tabu Search. These both metaheuristics are two of the most used ones along the history for solving optimization problems. In this first study, the comparison is performed for the well-known Traveling Salesman Problem.
\end{abstract}

\section{Keywords}

Golden Ball; Simulated Annealing; Tabu Search; Traveling Salesman Problem; TSP

\section{INTRODUCTION}

Golden Ball (GB) is a multiple-population meta-heuristic based on soccer concepts. The first complete version of the GB and its capacity to solve complex problems was presented in 2014 [3]. Until now, it has been tested with different kind of problems, such as the symmetric and asymmetric traveling salesman problem (TSP), N-Queens, the one dimensional bin packing problem, and various sorts of vehicle routing problems and rich vehicle routing problems $[4]$.

Permission to make digital or hard copies of part or all of this work for personal or classroom use is granted without fee provided that copies are not made or distributed for profit or commercial advantage and that copies bear this notice and the full citation on the first page. Copyrights for third-party components of this work must be honored. For all other uses, contact the owner/author(s).

GECCO'16 Companion July 20-24, 2016, Denver, CO, USA

(C) 2016 Copyright held by the owner/author(s).

ACM ISBN 978-1-4503-4323-7/16/07.

DOI: http://dx.doi.org/10.1145/2908961.2931634
In line with this, until now, the GB has only been compared with few techniques, such as different kind of Genetic Algorithms and Distributed Genetic Algorithms. In this way, the main motivation of this work is to prove that the GB can also compete with other famous and widely used techniques in terms of results quality. To perform this task, in this study, the results obtained by the GB are compared with the ones obtained by two different and frequently used meta-heuristics: the Evolutionary Simulated Annealing (ESA) [8] and the Tabu Search (TS) [1]. In this first study, the well-known TSP is used for the comparison.

The remainder of this work is structured as follows: in Section 2 the main concepts of the GB are briefly described. The paper continues with the experimentation in Section 3, and finishes with the main conclusions in Section 4.

\section{GOLDEN BALL METAHEURISTIC}

The GB is a recently proposed meta-heuristic based on soccer concepts. In this paper, the main concepts of the technique are shortly described. For further information about this meta-heuristic, the reading of [3] is highly recommended.

As can be read in the previous section, the GB is a multiple-population based meta-heuristic. First, in the initialization phase, the whole population of solutions (called players) is randomly created. Then, these players are randomly divided among a fixed number of subpopulations (called teams). Each team has its own training method (or coach), which is randomly assigned in this first phase. This training is the way in which each player in the team individually evolves along the execution. One training function for the TSP could be, for example, the widely used 2-opt [2]. Another important training is the called Custom Training. In this workout, a player which is trapped in a local optimum receives a special training in cooperation with the best player of its team. One possible custom training function could be, for example, the famous Order Crossover.

Once the initialization phase is finished, the competition phase starts. This second step is divided in seasons, composed by weeks. Every week all the teams train 
independently, and they face each other creating a league. At the end of every season, a transfer procedure takes place. In this procedure the players and coaches can switch teams. The competition phase is repeated iteratively until the termination criterion is reached.

\section{EXPERIMENTATION}

All the tests conducted in this work have been performed on an Intel Core i5 - 2410 laptop, with $2.30 \mathrm{GHz}$ and a RAM of 4 GB. Java has been used as the programming language. In these preliminary tests, 16 instances have been used with 50 to 144 nodes, which have been obtained from the TSPLIB Benchmark [6]. For this experimentation, the implementations described in [5] and [7] have been used for the ESA and TS, respectively. Every instance has been run 30 times, and each one has a number in its name which represents the number of nodes it has. The obtained results are shown in Table 1. In this Table, the results average, standard deviation and average runtime (in seconds) are shown. It is important to highlight that, as far as possible, the same parameterization has been used for all the techniques, in terms of population size, termination criterion, movement functions. . . In this way, the comparison can be as fair as possible.

Table 1: Results of the ESA, TS and GB for the TSP.

\begin{tabular}{|l||rrr||rrr||rrr|}
\hline Instance & \multicolumn{3}{|c||}{ ESA } & \multicolumn{3}{|c||}{ TS } & \multicolumn{3}{c|}{ GB } \\
\hline \hline Name & Avg. S. dev. Time & \multicolumn{3}{|c|}{ Avg. S. dev. Time } & \multicolumn{2}{|c}{ Avg. S. dev. Time } \\
\hline Eilon50 & 427.6 & 1.7 & 1.5 & 427.8 & 1.6 & 1.4 & $\mathbf{4 2 7 . 2}$ & 1.5 & 1.1 \\
Eil51 & 431.6 & 2.9 & 1.7 & 428.9 & 1.4 & 1.5 & $\mathbf{4 2 8 . 6}$ & 1.3 & 1.4 \\
Berlin52 & $\mathbf{7 5 4 2 . 0}$ & 0.0 & 2.1 & $\mathbf{7 5 4 2 . 0}$ & 0.0 & 2.2 & $\mathbf{7 5 4 2 . 0}$ & 0.0 & 2.1 \\
St70 & $\mathbf{6 7 9 . 1}$ & 2.8 & 3.9 & 679.2 & 3.4 & 4.1 & 679.4 & 3.5 & 4.2 \\
Eilon75 & 550.2 & 3.9 & 4.5 & 552.3 & 3.7 & 4.7 & $\mathbf{5 4 4 . 3}$ & 3.3 & 5.4 \\
Eil76 & 553.7 & 4.2 & 5.1 & $\mathbf{5 4 5 . 1}$ & 3.4 & 5.4 & 547.3 & 3.7 & 5.5 \\
KroA100 & 21481.7 & 150.1 & 10.6 & 21495.4 & 120.9 & 10.1 & $\mathbf{2 1 3 8 6 . 7}$ & 99.7 & 9.5 \\
KroB100 & 22602.2 & 210.2 & 11.1 & 22612.8 & 205.8 & 10.7 & $\mathbf{2 2 3 1 1 . 0}$ & 139.6 & 9.7 \\
KroC100 & 21170.4 & 188.7 & 12.0 & 21192.6 & 166.6 & 12.3 & $\mathbf{2 0 9 6 8 . 2}$ & 111.3 & 9.3 \\
KroD100 & 21726.5 & 156.9 & 11.7 & 21761.3 & 155.4 & 11.2 & $\mathbf{2 1 4 8 5 . 8}$ & 188.2 & 9.7 \\
KroE100 & 22499.7 & 171.4 & 11.4 & 22534.8 & 175.5 & 10.9 & $\mathbf{2 2 2 6 6 . 8}$ & 158.1 & 9.8 \\
Eil101 & 656.4 & 4.4 & 11.7 & 658.0 & 5.1 & 11.4 & $\mathbf{6 4 5 . 7}$ & 4.3 & 8.9 \\
Pr107 & 44821.5 & 179.3 & 12.1 & 44834.3 & 227.0 & 11.8 & $\mathbf{4 4 6 9 3 . 0}$ & 210.7 & 10.1 \\
Pr124 & 59593.6 & 367.8 & 18.5 & 59560.4 & 453.1 & 17.9 & $\mathbf{5 9 3 4 8 . 2}$ & 190.3 & 16.2 \\
Pr136 & 99864.4 & 655.7 & 23.4 & 99802.3 & 914.2 & 23.0 & $\mathbf{9 8 9 0 6 . 5}$ & 986.7 & 23.7 \\
Pr144 & 58807.3 & 220.9 & 33.9 & 58973.1 & 762.4 & 32.7 & $\mathbf{5 8 7 1 2 . 0}$ & 247.7 & 34.1 \\
\hline
\end{tabular}

Some preliminary conclusions can be drawn from these results. The most important one is that the GB obtains better results than the ESA and TS in the $81.25 \%$ (13 out of 16) of the instances. Additionally, the GB only gets worse results in 2 of the 16 instances (st70 and Eil76). On the other hand, in terms of runtime, the three techniques perform in a similar way, being the GB slightly better.

Another important feature that is obligatory to highlight is the robustness of the GB. The standard deviation of the results obtained by the GB is lower than the deviation of other algorithms. This means that for the GB, the differences between the worst and the best results of every instance are not very large. This characteristic gives robustness and reliability to the algorithm, something important whether the technique will be used in a real environments.
In general, it can be firmly concluded that the GB is a promising technique in comparison also with the ESA and TS. From this point of view, it can be said that the principal objective of this research has been met.

\section{CONCLUSIONS}

In this study, the performance of the Golden Ball has been compared with the ones obtained by an Evolutionary Simulated Annealing and a Tabu Search for the Traveling Salesman Problem. The main motivation of this first study is to confirm that the GB can compete with some of the most used techniques of the literature, such as the ESA and the TS. In this first study, the TSP has been used for the comparison, and looking at the obtained results, it can be concluded that the main objective has been met. As future work, a more thorough experimentation has been planned, performing statistical tests, using additional meta-heuristics and problems for the comparison, as well as new instances for the TSP.

\section{ACKNOWLEDGMENTS}

This project was supported by the European Unions Horizon 2020 research and innovation programme through the 636220 project. As well as by the projects TEC201345585-C2-2-R from the Spanish Ministry of Economy and Competitiveness.

\section{REFERENCES}

[1] F. Glover. Tabu search-part i. ORSA Journal on computing, 1(3):190-206, 1989.

[2] S. Lin. Computer solutions of the traveling salesman problem. The Bell System Technical Journal, 44(10):2245-2269, 1965.

[3] E. Osaba, F. Diaz, and E. Onieva. Golden ball: a novel meta-heuristic to solve combinatorial optimization problems based on soccer concepts. Applied Intelligence, 41(1):145-166, 2014.

[4] E. Osaba, F. Diaz, E. Onieva, P. López-García, R. Carballedo, and A. Perallos. A parallel meta-heuristic for solving a multiple asymmetric traveling salesman problem with simultaneous pickup and delivery modeling demand responsive transport problems. In Hybrid Artificial Intelligent Systems, pages 557-567. Springer, 2015.

[5] E. Osaba, X.-S. Yang, F. Diaz, P. Lopez-Garcia, and R. Carballedo. An improved discrete bat algorithm for symmetric and asymmetric traveling salesman problems. Engineering Applications of Artificial Intelligence, 48:59-71, 2016.

[6] G. Reinelt. TSPLIB-a traveling salesman problem library. ORSA journal on computing, 3(4):376-384, 1991.

[7] J. J. Schneider and S. Kirkpatrick. Tabu search applied to tsp. Stochastic Optimization, pages 441-447, 2006.

[8] P. P. Yip and Y.-H. Pao. Combinatorial optimization with use of guided evolutionary simulated annealing. IEEE Transactions on Neural Networks, 6(2):290-295, 1995. 